\title{
COEXTENSIONS OF REGULAR SEMIGROUPS BY RECTANGULAR BANDS. II
}

\author{
BY
}

\author{
JOHN MEAKIN $^{1}$ AND K. S. S. NAMBOORIPAD ${ }^{2}$
}

\begin{abstract}
A construction of all coextensions of a regular semigroup $S$ by rectangular bands is obtained. The construction is analogous to Hall's construction of orthodox semigroups as spined products of bands and inverse semigroups and reduces to that construction when $S$ is inverse. The results are specialized to provide a construction of the category of all normal coextensions of a regular semigroup.
\end{abstract}

If $S^{\prime}$ is a regular semigroup, then by a coextension of $S^{\prime}$ we mean a pair $(S, \phi)$ where $S$ is a regular semigroup and $\phi$ is a homomorphism from $S$ onto $S^{\prime}:(S, \phi)$ is called a coextension of $S^{\prime}$ by rectangular bands if, for each $e^{\prime} \in E\left(S^{\prime}\right), \phi^{-1}\left(e^{\prime}\right)$ is a rectangular subband of $S$. If $S^{\prime}$ is a regular semigroup and $\theta: E \rightarrow E\left(S^{\prime}\right)$ is a bimorphism, then by an extension of $\theta$ by $S^{\prime}$ we mean a pair $(S, \phi)$ where $S$ is a regular semigroup with $E(S)=E$ and $\phi: S \rightarrow S^{\prime}$ is a surjective homomorphism with $E(\phi)=\theta$. In [5] the authors have obtained a construction of all coextensions of a biordered set $E^{\prime}$ by rectangular biordered sets; that is, all bimorphisms $\theta: E \rightarrow E^{\prime}$ such that the congruence classes of $\operatorname{ker} \theta$ are rectangular. In this paper we shall describe all extensions of such bimorphisms which give rise to coextensions of regular semigroups by rectangular bands. Our construction is analogous to Hall's construction [3] of orthodox semigroups as spined products of bands and inverse semigroups.

1. Preliminary results, inductive groupoids. We assume familiarity with the basic ideas and notation of semigroup theory as presented in the book of Clifford and Preston [1]. In addition we assume the definitions, results and notation of our paper [5] and we shall not repeat these results here.

By a groupoid we mean a small category in which every morphism is an isomorphism: as in [6] we denote the morphism set of a groupoid $G$ also by $G$; the vertex set (set of objects) will be denoted by $V(G)$. A groupoid $G$ together with a partial order $\leqslant$ on $G$ is called an ordered groupoid if it satisfies the conditions (0G1)-(0G3) of [6]: an inductive groupoid is a pair $(G, \varepsilon)$ consisting of an ordered

Received by the editors March 18, 1981.

1980 Mathematics Subject Classification. Primary 20M10.

Key words and phrases. Regular semigroup, inductive groupoid, coextension, rectangular band, spined product, normal mapping, functor, natural transformation.

${ }^{1}$ The first author's research was supported partially by a grant from the U. S. National Science Foundation and was done while he was a Visiting Professor in the Department of Mathematics, University of Kerala.

${ }^{2}$ The second author gratefully acknowledges financial assistance from the Indian University Grants Commission through University of Kerala towards completing the research project. 
groupoid $G$ and a $V$-isomorphism $\varepsilon: \mathcal{G}(E) \rightarrow G$ (where $E$ is a biordered set and $\mathcal{G}(E)$ is the groupoid of $E$-chains of $E$ ) which satisfies the conditions (IG1) and (IG2) of [6] and their duals. We refer the reader to $[6, \S 4]$ for a description of the category of inductive groupoids and its relation to the category of regular semigroups. As in [6], $G$ denotes the functor from the category of regular semigroups to the category of inductive groupoids and $S$ denotes the functor from the category of inductive groupoids to the category of regular semigroups. We identify each regular semigroup $S$ with $S(G(S))$ by the natural isomorphism $\eta_{S}$ and each inductive groupoid $G$ with $G(S(G))$ by the natural isomorphism $\nu_{G}$ (see [6]).

If $E$ is a biordered set we denote by $T^{*}(E)$ the groupoid of partial $\omega$-isomorphisms of $E . T^{*}(E)$ is an inductive groupoid relative to the evaluation $\tau$ (see [6]). As in [6], the domain of $\alpha \in T^{*}(E)$ is denoted by $e_{\alpha}$ and the range of $\alpha$ is denoted by $f_{\alpha}$; that is, $\alpha$ is a partial $\omega$-isomorphism from $\omega\left(e_{\alpha}\right)$ onto $\omega\left(f_{\alpha}\right)$. If $G$ is an inductive groupoid, $x \in G$ and $g \in \omega\left(e_{x}\right)$ we denote the restriction of $x$ to $g$ by $g * x$ (see [6]) and define $g a_{G}(x)=f_{g_{* x}}$. From [6, Proposition 3.8] we have the following result.

Proposition 1.1. Let $G$ be an inductive groupoid. Then $\mathfrak{a}_{G}(x): \omega\left(e_{x}\right) \rightarrow \omega\left(f_{x}\right)$ is an $\omega$-isomorphism of $E=V(G)$ and the map $\mathfrak{a}_{G}: x \rightarrow \mathfrak{a}_{G}(x)$ is an.inductive functor (with $\left.V\left(a_{G}\right)=\iota_{E}\right)$ of $G$ to $T^{*}(E)$ such that the following diagram commutes:

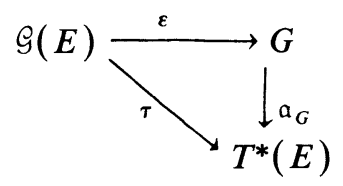

In particular, if $G$ is a full inductive subgroupoid of $T^{*}(E)$ then $\mathfrak{a}_{G}$ is the inclusion of $G$ in $T^{*}(E)$.

If $S$ is a regular semigroup and $\left(x, x^{\prime}\right) \in G(S)$ it is clear that $e_{\left(x, x^{\prime}\right)}=x x^{\prime}$ and $f_{\left(x, x^{\prime}\right)}=x^{\prime} x$. Also, for $g \in \omega\left(x x^{\prime}\right), g *\left(x, x^{\prime}\right)=\left(g x, x^{\prime} g\right)$ and so $f_{g_{*}\left(x, x^{\prime}\right)}=x^{\prime} g x$. Thus the $\omega$-isomorphism $\mathfrak{a}_{G(S)}\left(x, x^{\prime}\right)$ maps $g \in \omega\left(x x^{\prime}\right)$ to $x^{\prime} g x \in \omega\left(x^{\prime} x\right)$. We denote the homomorphism $S\left(\mathfrak{a}_{G}\right)$ of $S(G)$ into $S\left(T^{*}(E(S))\right)=T(E(S))$ by $\overline{\mathfrak{a}}_{S}$ (see [6]). From [6] we know that ker $\overline{\mathrm{a}}_{S}=\mu(S)$, the maximum indempotent-separating congruence on $S$, and that $S$ is fundamental if and only if $\overline{\mathrm{a}}_{\mathrm{S}}$ is an isomorphism of $S$ onto a full subsemigroup of $T(E(S))$.

Let $\theta: E \rightarrow E^{\prime}$ be a surjective bimorphism. An $\omega$-isomorphism $\alpha \in T^{*}(E)$ is compatible with $\theta$ if there exists $\alpha^{\prime} \in T^{*}\left(E^{\prime}\right)$ such that the following diagram commutes:

$$
\begin{array}{ccc}
\omega\left(e_{\alpha}\right) & \stackrel{\theta}{\rightarrow} & \omega\left(e_{\alpha^{\prime}}\right) \\
\downarrow \alpha & & \downarrow \alpha^{\prime} \\
\omega\left(f_{\alpha}\right) & \stackrel{\theta}{\rightarrow} & \omega\left(f_{\alpha^{\prime}}\right)
\end{array}
$$

that is, $(g \alpha) \theta=(g \theta) \alpha^{\prime}$ for all $g \in \omega\left(e_{\alpha}\right)$. If $\alpha$ is compatible with $\theta$, then it is easy to see that the $\omega$-isomorphism $\alpha^{\prime}$ making the above diagram commutative is unique, so 
that $\theta^{*}: \alpha \rightarrow \alpha^{\prime}$ is a mapping of the subset $\mathscr{T}_{\theta} \subseteq T^{*}(E)$ consisting of all $\omega$-isomorphisms compatible with $\theta$ into $T^{*}\left(E^{\prime}\right)$. We have the following:

Proposition 1.2 [6]. $\mathcal{T}_{\theta}$ is a full inductive subgroupoid of $T^{*}(E)$ and $\theta^{*}: \mathscr{丁}_{\theta} T^{*}(E)$ is an inductive functor with $V\left(\theta^{*}\right)=\theta$ such that, for all inductive functors $\phi: G \rightarrow G^{\prime}$ with $V(\phi)=\theta$, the following diagram commutes:

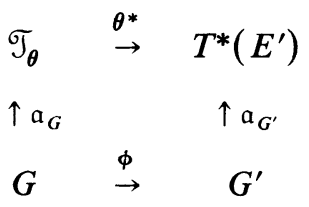

In particular if $\phi: \mathcal{T} \rightarrow T^{*}\left(E^{\prime}\right)$ with $\mathcal{T} \subseteq T^{*}(E)$ and $V(\phi)=\theta$, then $\mathcal{T} \subseteq \mathcal{T}_{\theta}$ and $\phi=\left.\theta^{*}\right|_{\text {g }}$.

The last statement of the proposition implies that $\mathscr{T}_{\theta}$ is the maximum subgroupoid of $T^{*}(E)$ to which $\theta$ extends as an inductive functor and that the extension is unique. Moreover, if $\phi: G \rightarrow G^{\prime}$ is any inductive functor such that $V(\phi)=\theta$, then $\operatorname{im}\left(a_{G}\right) \subseteq \mathcal{T}_{\theta}$ and $\phi$ is surjective if and only if $\operatorname{im}\left(a_{G^{\prime}}\right) \subseteq \operatorname{im} \theta^{*}$.

2. Coextensions of regular semigroups by rectangular bands. If $(S, \phi)$ is a coextension of $S^{\prime}$ by rectangular bands, it is clear that $(I(S), I(\phi))$ is a coextension of $I\left(S^{\prime}\right)$ by rectangular bands and the construction of such coextensions is described in Theorem 4.5 of [5]. (Here $I(S)$ denotes the subsemigroup of $S$ which is generated by its idempotents.) In particular, the induced coextension $(E(S), E(\phi))$ of the biordered set $E\left(S^{\prime}\right)$ must be of the form $E\left(E\left(S^{\prime}\right),\left\{E_{\alpha}\right\}, \phi, \psi\right)$ where the family of mappings $\phi$ and $\psi$ satisfies conditions (Pi), $i=1,2,3$, and (P4) of [5] relative to $I\left(S^{\prime}\right)$ (see $\left.[5, \S 4]\right)$. We shall say that $(E, \theta)$ is a strong coextension of $E^{\prime}$ relative to the idempotent-generated regular semigroup $S^{\prime}$ with $E\left(S^{\prime}\right)=E^{\prime}$ if the family of mappings $\phi$ and $\psi$ associated with $(E, \theta)$ satisfies $(\mathrm{P} 4)$ relative to $S^{\prime} .(E, \theta)$ is strong if it is strong relative to some $S^{\prime}$.

Let $(E, \theta)$ be a coextension of the biordered set $E^{\prime}$. By Proposition 1.2 and [6, Theorem 4.12], $S\left(\theta^{*}\right): S\left(\mathcal{T}_{\theta}\right) \rightarrow S\left(T^{*}\left(E^{\prime}\right)\right)=T\left(E^{\prime}\right)$ is a homomorphism such that $E\left(S\left(\theta^{*}\right)\right)=\theta$. We write $T_{\theta}=S\left(\mathcal{T}_{\theta}\right)$ and $\bar{\theta}=S\left(\theta^{*}\right)$. Thus $\operatorname{ker} \bar{\theta}$ is a congruence on $T_{\theta}$ such that $\operatorname{ker} \bar{\theta} \cap(E \times E)=\operatorname{ker} \theta$. In fact $\operatorname{ker} \bar{\theta}$ is the largest congruence with this property; the smallest congruence with this property is denoted by $\eta_{\theta}$. (Such a congruence $\eta_{\theta}$ exists by the results of Reilly and Scheiblich [8].)

Recall that, if $S$ is a regular semigroup, then $\rho(S)$ denotes the maximum congruence on $S$ whose congruence classes containing idempotents are rectangular bands [5]. The next proposition characterizes strong coextensions in terms of $\eta_{\theta}$.

Proposition 2.1. Let $(E, \theta)$ be a coextension of a biordered set $E^{\prime}$. Thus $\eta_{\theta} \subseteq \rho\left(T_{\theta}\right)$ if and only if $(E, \theta)$ is strong relative to some idempotent generated regular semigroup $S^{\prime}\left(\right.$ with $\left.E\left(S^{\prime}\right)=E^{\prime}\right)$.

Proof. Let $S_{\theta}$ denote the semigroup $T_{\theta} / \eta_{\theta}$ with $E\left(S_{\theta}\right)$ identified with $E^{\prime}$ by identifying $e \eta_{\theta}$ with $e \theta$. If $\eta \vec{\theta}$ is the canonical homomorphism of $T_{\theta}$ onto $S_{\theta}$, then the 
foregoing identification implies that $E\left(\eta_{\theta}\right)=\theta$. If $\eta_{\theta} \subseteq \rho\left(T_{\theta}\right)$, it is clear that $\left(T_{\theta}, \eta_{\theta}\right)$ is a coextension of $S_{\theta}$ by rectangular bands inducing $(E, \theta)$ and so $(E, \theta)$ is strong relative to $I\left(S_{\theta}\right)$.

Conversely assume that $(E, \theta)$ is a strong coextension of $E^{\prime}$ relative to some idempotent-generated regular semigroup $S^{\prime}$ with $E\left(S^{\prime}\right)=E^{\prime}$. Thus by Theorem 4.5 of [5] there exists a coextension $(S, p)$ of $S^{\prime}$ by rectangular bands inducing $(E, \theta)$ (that is, with $E(p)=\theta$ ) and with $S$ an idempotent-generated semigroup on $E$. If $\mu$ is the maximum idempotent-separating congruence on $S$, it follows by Proposition 2.13 of [5] that the congruence $\rho=(\operatorname{ker} p \circ \mu) / \mu$ is contained in $\rho(I(E)$ ) (where $I(E)$ denotes the fundamental idempotent-generated semigroup on $E$ ). Now $\mu=\operatorname{ker} \overline{\mathfrak{a}}_{S}$ and $E\left(\overline{\mathfrak{a}}_{S}\right)=\iota_{E}$, so $\rho \cap(E \times E)=\operatorname{ker} \theta$. So, if $e \in E$ and $e, f \in E_{e^{\prime}}=e^{\prime} \theta^{-1}$ with

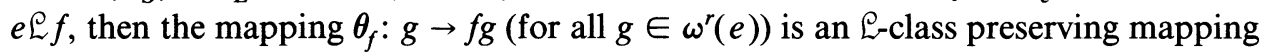
of $\omega^{r}(e)$ onto $\omega^{r}(f)$ satisfying conditions (1) and (2) of Proposition 2.8 of [5] in $I(E)$. Since $I(E) \subseteq T_{\theta}$, it follows that the condition (1) holds in $T_{\theta}$ also. To prove (2), we first note that for all $\left(x, x^{\prime}\right) \in G\left(T_{\theta}\right)$ there exists a unique $\alpha \in \mathcal{T}_{\theta}$ such that $\nu_{G}\left(\underline{\alpha)}=\left(x, x^{\prime}\right)\right.$ where $\nu_{G}: \sigma_{\theta} \rightarrow G\left(T_{\theta}\right)$ is the natural isomorphism sending $\alpha$ to $\left(\bar{\alpha}, \alpha^{-1}\right)$ (see [6]). By the convention adopted (see $\S 1$ ) we identify $\alpha$ with $\left(\bar{\alpha}, \overline{\alpha^{-1}}\right)$. Since $V\left(G\left(T_{\theta}\right)\right)=E\left(T_{\theta}\right)$ by the definition of $G\left(T_{\theta}\right)$ (see [6]), the identification above also identifies $e \in E^{\prime}$ with the corresponding idempotent $\bar{e}$ in $E\left(T_{\theta}\right)$. Hence for $g \in \omega\left(e_{\alpha}\right)$,

$$
g \alpha=f_{g_{*} \alpha}=f_{g_{*}\left(x, x^{\prime}\right)}=g a\left(x, x^{\prime}\right)=x^{\prime} g x
$$

(see Proposition 1.1). Suppose that $h \in S\left(f_{\alpha}, e\right)$. By $(1), f h \in S\left(f_{\alpha}, f\right)$ and $h, h f \in$ $E_{h \theta}$. Therefore $f_{\alpha} h, f_{\alpha}(f h) \in E_{\left(f_{\alpha} h\right) \theta}$ and $f_{\alpha} h \mathcal{L} f_{\alpha}(f h)$. Moreover, $x h x^{\prime}=x f_{\alpha} h x^{\prime}=$ $\left(f_{\alpha} h\right) \alpha^{-1}, x f h x^{\prime}=\left(f_{\alpha}(f h)\right) \alpha^{-1}$. Since $\alpha \in \mathcal{F}_{\theta}$, it follows that $x h x^{\prime}, x(f h) x^{\prime} \in$ $E_{\left(\left(f_{\alpha} h\right) \alpha^{-1}\right) \theta}$ which is a $\rho$-class in $I(E)$. Hence we have $V\left(x h x^{\prime}\right)=V\left(x(f h) x^{\prime}\right)=$ $V\left(x\left(\theta_{f} h\right) x^{\prime}\right)$ in $I(E)$. But $I(E)$ is a full subsemigroup of $T_{\theta}$ and so this equality holds in $T_{\theta}$ also. Thus $\theta_{f}$ satisfies condition (2) in $T_{\theta}$ and so by Proposition 2.8 of [5], $(e, f) \in \rho\left(T_{\theta}\right)$. If $e^{\Re} f$ and $e, f \in E_{e^{\prime}}$, we can prove dually that $(e, f) \in \rho\left(T_{\theta}\right)$. Therefore, for all $e, f \in E_{e^{\prime}}$ we have $(e, f) \in \rho\left(T_{\theta}\right)$. Hence $\eta_{\theta} \cap \rho\left(T_{\theta}\right)=\rho_{1}$ is a congruence on $T_{\theta}$ such that $e \rho_{1}=E_{e \theta}$ for all $e \in E$; that is, $\rho_{1} \cap E \times E=\operatorname{ker} \theta$. But $\eta_{\theta}$ is the minimum congruence with this property and hence $\rho_{1}=\eta_{\theta} \subseteq \rho\left(T_{\theta}\right)$, as required.

THEOREM 2.2. Let $U$ be a regular semigroup and $(E, \theta)$ be a strong coextension of $E^{\prime}=E(U)$. Let $\zeta: U \rightarrow S_{\theta}$ be a homomorphism with $E(\zeta)=\iota_{E^{\prime}}$. Then there exists a regular semigroup $S=S(U, \theta, \zeta)$ with $E(S)=E$ and a homomorphism $p: S \rightarrow U$ with $E(p)=\theta$ such that the following diagram is a pullback as well as a pushout in the category $\underline{R} \underline{S}$ (of regular semigroups).

$\begin{array}{lll}T_{\theta} & \stackrel{\eta \forall}{\rightarrow} & S_{\theta} \\ \uparrow \overline{\mathrm{a}}_{S} & & \uparrow \zeta \\ S & \stackrel{p}{\rightarrow} & U\end{array}$


$S$ is isomorphic to the spined product of $T_{\theta}$ and $U$ with respect to $\eta_{\theta}$ and $\zeta:(S, p)$ is a coextension of $U$ by rectangular bands.

Conversely if $(S, p)$ is a coextension of $U$ by rectangular bands, then $(E(S), E(p))$ is a strong coextension of $E(U)$ and there exists a homomorphism $\zeta: U \rightarrow S_{E(p)}$ with $E(\zeta)=\iota_{E(U)}$ such that $S$ is isomorphic to $S(U, E(p), \zeta)$.

Proof. Let $S^{\prime}$ be the spined product of $T_{\theta}$ and $U$ with respect to $\eta \vec{\theta}$ and $\zeta$, that is

$$
S^{\prime}=\left\{(x, u) \in T_{\theta} \times U: x \eta_{\theta}^{*}=u \zeta\right\}
$$

with product defined by $(x, u)(y, v)=(x y, u v)$. If $(x, u) \in S^{\prime}$ and $x^{\prime} \in V(x)$, then $x^{\prime} \eta^{*} \in V(u \zeta)$ and by Lemma 1 of Hall [4] there exists $u^{\prime} \in V(u)$ such that $u^{\prime} \zeta=x^{\prime} \eta_{\theta}$ and so $\left(x^{\prime}, u^{\prime}\right) \in V((x, u))$ in $S^{\prime}$. Hence $S^{\prime}$ is regular. Clearly, $(x, u) \in$ $E\left(S^{\prime}\right)$ iff $x \in E\left(T_{\theta}\right)=E$ and $u=x \theta$; also, $e \rightarrow(e, e \theta)$ is an isomorphism of $E$ onto $E\left(S^{\prime}\right)$. Let $S=S(u, \theta, \zeta)$ be the semigroup obtained by identifying $E\left(S^{\prime}\right)$ with $E$ by the isomorphism $(e, e) \rightarrow e$. If $p$ is the homomorphism $(x, u) \rightarrow u$, then it is onto. For, if $u \in U, u \zeta \in S_{\theta}$ and since $\eta_{\theta}$ is onto, there exists $x \in T_{\theta}$ with $x \eta_{\theta}=u \zeta$ and so $(x, u) \in S$ and $(x, u) p=u$. Also, $p$ is one-to-one on $\mathcal{F G}$-classes. For if $(x, u) \mathcal{H}(y, u)$, then $x \eta_{\theta}=y \eta_{\theta}$ and since $\eta_{\theta} \subseteq \rho\left(T_{\theta}\right)$, and since $x \mathcal{H} y$ in $T_{\theta}$, we have $x=y$. Let $p^{\prime}$ denote the projection $(x, u) \rightarrow x$. Thus $(x, u) p^{\prime} \eta_{\theta}=(x, u) p \zeta$; that is $p^{\prime} \eta_{\theta}=p \zeta$. Since we have identified $(e, e \theta)$ with $e$, it follows that $E\left(p^{\prime}\right)=\iota_{E}$. Since $E$ : $S \rightarrow E(S)$ is a functor, we have,

$$
\theta=\iota_{E} \theta=E\left(p^{\prime}\right) E(\eta \forall)=E(p) E(\zeta)=E(p) .
$$

This together with the fact that $p$ is one-to-one on $\mathcal{H}$-classes implies that $(S, p)$ is a coextension of $U$ by rectangular bands inducing $(E, \theta)$.

To show that $p^{\prime}=\overline{\mathfrak{a}}_{S}$, consider $(x, u) \in S$ and $\left(x^{\prime}, u^{\prime}\right) \in V((x, u))$. Thus $x^{\prime} \in$ $V(x)$ in $T_{\theta}$ and so we may choose $\alpha \in \mathcal{J}_{\theta}$ such that $\bar{\alpha}=x, \overline{\alpha^{-1}}=x^{\prime}$. Let $g \in \omega\left(e_{\alpha}\right)$ : by identifying $\mathcal{T}_{\theta}$ and $G\left(T_{\theta}\right)$ we have (as in the proof of Proposition 2.1),

$$
g \alpha=g a\left(\bar{\alpha}, \overline{\alpha^{-1}}\right)=\overline{\alpha^{-1}} g \bar{\alpha}=x^{\prime} g x,
$$

and so

$$
(g \alpha) \theta=\left(x^{\prime} g x\right) \eta_{\theta}^{*}=u^{\prime} \zeta g \theta u \zeta=\left(u^{\prime} g \theta u\right) \zeta=u^{\prime} g \theta u
$$

since $E(\zeta)=\iota_{E^{\prime}}$. Also, in $S$, we have

$$
g a_{S}\left((x, u),\left(x^{\prime}, u^{\prime}\right)\right)=\left(x^{\prime} g x, u^{\prime}(g \theta) u\right)=(g \alpha,(g \alpha) \theta)=g \alpha
$$

and hence $a_{S}\left((x, u),\left(x^{\prime}, u^{\prime}\right)\right)=\alpha$. Therefore

$$
(x, u) \overline{\mathrm{a}}_{S}=\overline{\mathrm{a}_{S}\left((x, u),\left(x^{\prime}, u^{\prime}\right)\right)}=\bar{\alpha}=x=(x, u) p^{\prime} ;
$$

that is, $p^{\prime}=\overline{\mathrm{a}}_{S}$.

The fact that $S$ is isomorphic to the spined product implies that the diagram (2.1) is a pullback. To prove that it is a pushout, consider the commutative diagram:

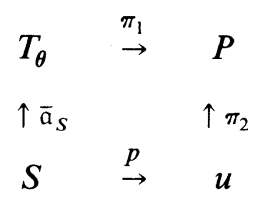


Thus $\psi_{1}=\theta \psi_{2}$ where $\psi_{i}=E\left(\pi_{i}\right), i=1,2$. Therefore $\operatorname{ker} \theta \subseteq \operatorname{ker} \psi_{1}$. If $(x, y) \in \eta_{\theta}$, then $V(x)=V(y)$ and so by Lemma 2.1 of [5], for $e \in E\left(R_{x}\right)$ and $f \in E\left(L_{x}\right)$, there exists $e^{\prime} \in E\left(R_{y}\right) \cap L_{e}$ and $f^{\prime} \in E\left(L_{y}\right) \cap R_{f}$ such that $y=e^{\prime} x f^{\prime}$. Now $e, e^{\prime} \in E_{e \theta}$ and so $e \psi_{1}=e^{\prime} \psi_{1}$. Hence $x=e x \operatorname{ker} \pi_{1} e^{\prime} x$. Similarly $e^{\prime} x \operatorname{ker} \pi_{1} e^{\prime} x f^{\prime}$ and so $(x, y) \in$ $\operatorname{ker} \pi_{1}$. Thus $\eta_{\theta} \subseteq \operatorname{ker} \pi_{1}$ and so $\pi$, defined by

$$
z \pi=x \pi_{1}
$$

for $z \in S_{\theta}$ and $x \in T_{\theta}$ with $x \eta_{\theta}=z$, is a single valued homomorphism such that $\pi_{1}=\eta \eta_{\theta} \pi$. To prove that $\zeta \pi=\pi_{2}$, consider $u \in U$. Thus there exists $x \in S$ such that $u=x p$ and so

$$
u \zeta \pi=(x p \zeta) \pi=\left(x \overline{\mathrm{a}}_{S} \eta \forall\right) \pi=\left(x \overline{\mathrm{a}}_{S}\right) \pi_{1}=(x p) \pi_{2}=u \pi_{2} .
$$

Thus $\zeta \pi=\pi_{2}$ and this proves that the diagram (2.1) is a pushout in $R S$.

Conversely assume that $(S, p)$ is a coextension of $U$ by rectangular bands. Write $E(S)=E, E(p)=\theta$ and $E^{\prime}=E(U)$. Then, clearly, $(E, \theta)$ is a strong coextension of $E^{\prime}$ with respect to $I(E)$. Define $\zeta: U \rightarrow S_{\theta}$ by $u \zeta=\left(x \overline{\mathfrak{a}}_{S}\right) \eta \eta_{\theta}$ for some $x \in S$ with $x p=u$. This definition is single valued. For if $x p=y p$, then $(x, y) \in \operatorname{ker} p$. Hence $\left(x \overline{\mathfrak{a}}_{S}, y \overline{\mathfrak{a}}_{S}\right) \in \rho$ where $\rho$ is the image of the congruence ker $p \circ \mu$ under $\overline{\mathfrak{a}}_{\mathrm{s}}$. Since $\operatorname{ker} \bar{a}_{S}=\mu$, by Proposition 2.13 of [5], $\rho \subseteq \rho\left(T_{\theta}\right)$. Since $E\left(\bar{a}_{S}\right)=\iota_{E}, E(p)=\theta$, it follows that $\rho \cap E \times E=\operatorname{ker} \theta$. Since $\eta_{\theta} \subseteq \rho\left(T_{\theta}\right)$ and $E\left(\eta_{\theta}\right)=\theta$, it follows that $\eta_{\theta}=\rho$. Hence $\left(x \overline{\mathrm{a}}_{S}\right) \eta_{\theta}=\left(y \overline{\mathrm{a}}_{S}\right) \eta$. To prove that $\zeta$ is a homomorphism, consider $u$, $v \in U$. If $x p=u$ and $y p=v$, then

$$
(u v) \zeta=((x y) p) \zeta=\left((x y) \overline{\mathfrak{a}}_{S}\right) \eta_{\theta}=\left(x \overline{\mathrm{a}}_{S}\right) \eta_{\theta}\left(y \overline{\mathrm{a}}_{S}\right) \eta_{\theta}=(u \zeta)(v \zeta) .
$$

Also, since $\overline{\mathfrak{a}}_{S} \eta \bar{\theta}=p \zeta$,

$$
\theta E(\zeta)=E\left(\overline{\mathrm{a}}_{S}\right) E\left(\eta \frac{\eta_{\theta}}{}\right)=\iota_{E} E\left(\eta_{\theta}^{\#}\right)=E\left(\eta_{\theta}^{\#}\right)=\theta
$$

and so $E(\zeta)=\iota_{E^{\prime}}$.

To prove that $S$ is isomorphic to $S(U, \theta, \zeta)$, define $t$ by

$$
x t=\left(x \overline{\mathfrak{a}}_{S}, x p\right) .
$$

Clearly, $t$ is a homomorphism of $S$ into $S(U, \theta, \zeta)$. If $x t=y t$, then $x \overline{\mathfrak{a}}_{S}=y \overline{\mathfrak{a}}_{S}$ and so $x \mathcal{H} y$ in $S$. Since $x p=y p$ and $p$ is one-to-one on $\mathcal{G}$-classes, we have $x=y$. Thus $t$ is one-to-one. To prove that $t$ is onto, consider $(z, u) \in S(U, \theta, \zeta)$. Since $p$ is onto, there exists $x \in S$ such that $x p=u$ and so $\left(x \overline{\mathrm{a}}_{S}\right) \eta \bar{\theta}=u \zeta=z \eta \bar{\theta}$. Since $\eta_{\theta} \subseteq \rho\left(T_{\theta}\right)$, we have $V\left(x \overline{\mathrm{a}}_{S}\right)=V(z)$ in $T_{\theta}$. Therefore, by Lemma 2.1 of [5], there exists $e \in E\left(R_{z}\right)$ and $f \in E\left(L_{z}\right)$ such that $e\left(x \bar{a}_{S}\right) f=z$. Since $E\left(\bar{a}_{S}\right)=\iota_{E}$, we have $($ exf $) \overline{\mathfrak{a}}_{S}=z$. Now $e \theta \in E\left(R_{u \zeta}\right)$ and $f \theta \in E\left(L_{u \zeta}\right)$ and since $E(\zeta)=\iota_{E^{\prime}}$, we have $u \in R_{e \theta} \cap L_{f \theta}$. Hence

$$
(e x f) p=(e p)(x p)(f p)=(e \theta) u(f \theta)=u .
$$

Therefore $(z, u)=\left((\operatorname{exf}) \overline{\mathrm{a}}_{S},(\operatorname{exf}) p\right)=(\operatorname{exf}) t$. This completes the proof.

REMARK. The hypothesis that $E(\zeta)=\iota_{E^{\prime}}$ in the statement of Theorem 2.2 is only a matter of convenience; the essential requirement here is that $E(\zeta)$ be an isomorphism.

REMARK. If $(E, \theta)$ is a coextension of a semilattice $E^{\prime}$, then it can be seen that every $\omega$-isomorphism of $E$ is compatible with $\theta$ so that, in this case, $T_{\theta}=T(E)$. 
Since strong coextensions of semilattices are bands, it follows that the foregoing theorem is exactly equivalent to Hall's spined product theorem for orthodox semigroups (Hall [3, Theorem 1]).

REMARK. Given a regular semigroup $S^{\prime}$ and a strong coextension $(E, \theta)$ of $E\left(S^{\prime}\right)$ relative to $I\left(S^{\prime}\right)$, it may not be possible to find a coextension of $S^{\prime}$ by rectangular bands inducing $(E, \theta)$, since there may not exist a homomorphism $\zeta$ of $S^{\prime}$ to $S_{\theta}$ so that $E(\zeta)$ is an isomorphism. The example given by Hall in [3] proves this (cf. $[3, \S 4.1])$.

We proceed to give an alternate description of the semigroup $S(U, \theta, \zeta)$ similar to Hall's second theorem of [3]. Let $(E, \theta)=E\left(E^{\prime},\left\{E_{e^{\prime}}\right\}, \phi, \psi\right)$. For $e^{\prime} \in E^{\prime}$ and $e \in E$, write

$$
E_{e^{\prime}}=I_{r\left(e^{\prime}\right)} \times \Lambda_{l\left(e^{\prime}\right)} \text { and } e=\left(i_{r(e)}, \lambda_{l(e)}\right) .
$$

It is clear that the mapping $R_{e} \rightarrow i_{r(e)}$ is a bijection of $T_{\theta} / \mathcal{R}$ onto

$$
I=\bigcup\left\{I_{r\left(e^{\prime}\right)}: e^{\prime} \in E^{\prime}\right\},
$$

so that we may define a partial order on $I$ making it a regular partially ordered set (cf. Grillet [2] and Nambooripad [7]). Similarly $L_{e} \rightarrow \lambda_{l(e)}$ is a bijection of $T_{\theta} / \mathcal{L}$ onto $\Lambda=\cup\left\{\Lambda_{l\left(e^{\prime}\right)}: e^{\prime} \in E^{\prime}\right\}$ and so $\Lambda$ is a regular partially ordered set with respect to the obvious partial order in $\Lambda$.

Now suppose that

$$
S^{\prime}=\left\{(u, i, \lambda): u \in U, i \in I_{r\left(u u^{\prime}\right)}, \lambda \in \Lambda_{l\left(u^{\prime} u\right)} \text { for } u^{\prime} \in V(u)\right\} .
$$

It is clear that $\pi:(\bar{\alpha}, u) \rightarrow\left(u, i_{r\left(e_{\alpha}\right)}, \lambda_{l\left(f_{\alpha}\right)}\right)$ is a mapping of $S(U, \theta, \zeta)$ into $S^{\prime}$. If $\pi(\bar{\alpha}, u)=\pi(\bar{\beta}, v)$, then clearly $u=v$ and $\bar{\alpha}$ and $\bar{\beta}$ are $\mathcal{H}$-equivalent in $T_{\theta}$. But since $(\bar{\alpha}, u),(\bar{\beta}, v) \in S, \bar{\alpha} \eta \eta_{\theta}=\bar{\beta} \eta \frac{H}{\theta}$ and since $\eta_{\theta}^{\#}$ is one-to-one on $\mathcal{G}$-classes, it follows that $\bar{\alpha}=\bar{\beta}$. If $(u, i, \lambda) \in S^{\prime}$, where $i \in I_{r\left(e^{\prime}\right)}$ and $\lambda \in \Lambda_{l\left(f^{\prime}\right)}$ with $e^{\prime} \in E\left(R_{u}\right)$ and $f^{\prime} \in$ $E\left(L_{u}\right)$, we can find $e \in E_{e^{\prime}}$ and $f \in E_{f^{\prime}}$ such that $i_{r(e)}=i$ and $\lambda_{l(f)}=\lambda$. So there exists a unique $\bar{\alpha} \in R_{e} \cap L_{f}$ in $T_{\theta}$ such that $\bar{\alpha} \eta \bar{\theta}=u \zeta$. Thus $(\bar{\alpha}, u) \pi=(u, i, \lambda)$. This shows that the semigroup $S$ may be coordinatized in terms of $I, \Lambda$ and $U$ as above.

To describe the multiplication in $S^{\prime}$, first consider $(u, i, \lambda) \in S^{\prime}$. Let $\alpha \in \mathcal{T}_{\theta}$ be such that $(\bar{\alpha}, u) \pi=(u, i, \lambda)$. Let $\lambda_{\alpha^{-1}}$ be the mapping of $T_{\theta} / \mathcal{R}$ into itself defined by

$$
\lambda_{\alpha^{-1}}\left(R_{f}\right)=R_{(f \alpha h) \alpha^{-1}}
$$

where $h \in S\left(f_{\alpha}, f\right)$. Thus $\lambda_{\alpha^{-1}}$ is a normal mapping of $T_{\theta} / \mathcal{R}$ such that $R_{f_{\alpha}} \in M\left(\lambda_{\alpha^{-1}}\right)$ and $\operatorname{im} \lambda_{\alpha^{-1}}=T_{\theta} / \Re\left(R_{e_{\alpha}}\right)$ (the principal ideal generated by $R_{e_{\alpha}}$ ) (cf. Nambooripad [7, Theorem C]). Also, by Lemma 2.12 of [7], $\lambda_{\alpha^{-1}}$ is uniquely determined by $\bar{\alpha}$. Hence, $\lambda_{\alpha^{-1}}$ induces, in an obvious way, a normal mapping which we denote by $\Phi(u, i, \lambda)$ of $I$ such that $\operatorname{im} \Phi(u, i, \lambda)=I(i)$ and $M(\Phi(u, i, \lambda))=\{j \in I:(j, \lambda) \in$ $E\}$. Dually, there exists a normal mapping $\Psi(u, i, \lambda)$ of $\Lambda$ such that $\operatorname{im} \Psi(u, i, \lambda)=$ $\Lambda(\lambda)$ and $M(\Psi(u, i, \lambda))=\{\mu \in \Lambda:(i, \mu) \in E\}$.

Now let $(\bar{\alpha}, u) \pi=(u, i, \lambda)$ and $(\bar{\beta}, v) \pi=(v, j, \mu)$. If $(\bar{\alpha} \bar{\beta}, u v) \pi=(u v, k, l)$ then $k$ corresponds to $R_{\bar{\alpha} \bar{\beta}}$ and $l$ corresponds to $L_{\bar{\alpha} \bar{\beta}}$. But from [6] we know that $\bar{\alpha} \bar{\beta}=\overline{(\alpha \circ \beta)}_{h}$ where $h \in S\left(f_{\alpha}, e_{\beta}\right)$ and so $R_{\bar{\alpha} \bar{\beta}}=R_{e_{(\alpha \circ \beta) h}}$. Now, $e_{(\alpha \circ \beta)_{h}}=\left(f_{\alpha} h\right) \alpha^{-1}$ so that $R_{e_{(\alpha \circ \beta)_{h}}}=\lambda_{\alpha^{-1}}\left(R_{e_{\beta}}\right)$. Hence $k=\Phi(u, i, \lambda)(j)$. Dually $l=(\lambda) \Psi(v, j, \mu)$. 
Therefore if we define

$$
(u, i, \lambda)(v, j, \mu)=(u v, \Phi(u, i, \lambda)(j),(\lambda) \Psi(v, j, \mu))
$$

then with this product $S^{\prime}$ becomes a regular semigroup isomorphic to $S$. Thus we have the following theorem.

THEOREM 2.3. Let $U$ be a regular semigroup, $(E, \theta)$ be a strong coextension of $E^{\prime}=E(U)$ and $\zeta: U \rightarrow S_{\theta}$ be an idempotent separating homomorphism. Write $(E, \theta)=E\left(E^{\prime},\left\{E_{e^{\prime}}\right\}, \phi, \psi\right), \quad E_{e^{\prime}}=I_{r\left(e^{\prime}\right)} \times \Lambda_{l\left(e^{\prime}\right)}, \quad I=\bigcup\left\{I_{r\left(e^{\prime}\right)}: \quad e^{\prime} \in E^{\prime}\right\}, \quad \Lambda=$ $\cup\left\{\Lambda_{l\left(e^{\prime}\right)}: e^{\prime} \in E^{\prime}\right\}$ and for any $e \in E, e=\left(i_{r(e)}, \lambda_{l(e)}\right)$. Let

$$
S^{\prime}=\left\{(u, i, \lambda): i \in I_{r\left(u u^{\prime}\right)}, \lambda \in \Lambda_{l\left(u^{\prime} u\right)} \text { for } u^{\prime} \in V(u)\right\}
$$

and for each $(u, i, \lambda) \in S^{\prime}$, let $\Phi(u, i, \lambda)$ and $\Psi(u, i, \lambda)$ denote normal mappings of $I$ and $\Lambda$ respectively defined above. Then $S^{\prime}$, with product defined by

$$
(u, i, \lambda)(v, j, \mu)=(u v, \Phi(u, i, \lambda)(j),(\lambda) \Psi(v, j, \mu))
$$

is a regular semigroup and the mapping $p^{\prime}:(u, i, \lambda) \rightarrow u$ is a homomorphism of $S^{\prime}$ onto $U$ such that there exists an isomorphism $\chi: E \rightarrow E\left(S^{\prime}\right)$ making the following diagram commutative:

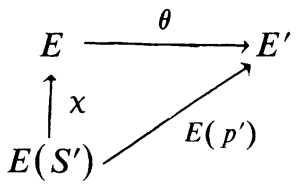

$\left(S^{\prime}, p^{\prime}\right)$ is a coextension of $U$ by rectangular bands.

Conversely every coextension of $U$ by rectangular bands can be (up to isomorphism) constructed in this way.

Let $(S, p)$ and $\left(S^{\prime}, p^{\prime}\right)$ be two coextensions of a regular semigroup $U$ by rectangular bands. A morphism $F:(S, p) \rightarrow\left(S^{\prime}, p^{\prime}\right)$ is a homomorphism $F: S \rightarrow S^{\prime}$ such that the following diagram commutes:

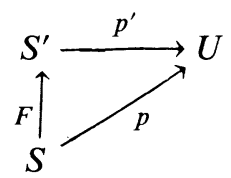

We denote by $\underline{R}(U)$ the category of all coextensions of a regular semigroup $U$ by rectangular bands in which morphisms are those defined above. Theorems 2.2 and 2.3 describe the construction of objects in $\underline{R}(U)$. We proceed to describe the construction of morphisms in this category. In the following we use the coordinatization of the coextension $(S, p)$, introduced in Theorem 2.3.

THeOREM 2.4. Let $(S, p)$ and $\left(S^{\prime}, p^{\prime}\right)$ be coextensions of a regular semigroup $U$ by rectangular bands. Let $I=S / \Re, I^{\prime}=S^{\prime} / \mathcal{R}, \Lambda=S / \mathcal{L}$ and $\Lambda^{\prime}=S^{\prime} / \mathcal{L}$. If $F:(S, p)$ $\rightarrow\left(S^{\prime}, p^{\prime}\right)$ is a morphism of coextensions, then the mappings $\theta_{R}: I \rightarrow I^{\prime}, \theta_{L}: \Lambda \rightarrow \Lambda^{\prime}$ defined by

$$
\theta_{R}\left(R_{x}\right)=R_{F(x)}, \quad \theta_{L}\left(L_{x}\right)=L_{F(x)}
$$


satisfy the following conditions: for all $(u, i, \lambda) \in S$ and $j \in I, \mu \in \Lambda$,

$$
\begin{aligned}
& \theta_{R}(\Phi(u, i, \lambda)(j))=\Phi\left(u, \theta_{R}(i), \theta_{L}(\lambda)\right)\left(\theta_{R}(j)\right), \\
& \theta_{L}(\Psi(u, i, \lambda)(\mu))=\Psi\left(u, \theta_{R}(i), \theta_{L}(\lambda)\right)\left(\theta_{L}(\mu)\right),
\end{aligned}
$$

where $\Phi(u, i, \lambda)[\Psi(u, i, \lambda)]$ denote the normal mapping of $I[\Lambda]$ defined in Theorem 2.3.

Conversely, given mappings $\theta_{R}: I \rightarrow I^{\prime}, \theta_{L}: \Lambda \rightarrow \Lambda^{\prime}$, satisfying the conditions above, then the mapping $F$ defined by

$$
F(u, i, \lambda)=\left(u, \theta_{R}(i), \theta_{L}(\lambda)\right)
$$

is a morphism of $(S, p)$ to $\left(S^{\prime}, p^{\prime}\right)$ such that

$$
\theta_{R}\left(R_{x}\right)=R_{F(x)}, \quad \theta_{L}\left(L_{x}\right)=L_{F(x)}
$$

for all $x \in S$.

Proof. Suppose that $F:(S, p) \rightarrow\left(S^{\prime}, p^{\prime}\right)$ is a morphism. If $F(u, i, \lambda)=\left(u^{\prime}, i^{\prime}, \lambda^{\prime}\right)$, then $i=R_{(u, i, \lambda)}, \quad \lambda=L_{(u, i, \lambda)}, i^{\prime}=R_{\left(u^{\prime}, i^{\prime}, \lambda^{\prime}\right)}=R_{F(u, i, \lambda)}$ and $\lambda^{\prime}=L_{F(u, i, \lambda)}$. Also $p^{\prime}(F(u, i, \lambda))=p^{\prime}\left(u^{\prime}, i^{\prime}, \lambda^{\prime}\right)=u^{\prime}$ and $p(u, i, \lambda)=u$. Hence $u=u^{\prime}$. Thus

$$
F(u, i, \lambda)=\left(u, \theta_{R}(i), \theta_{L}(\lambda)\right) \text {. }
$$

To prove the condition above, consider $j \in I$. Choose $\mu \in \Lambda$ and $\alpha \in E(U)$ so that $e=(\alpha, j, \mu) \in E(S)$. Then

$$
\begin{aligned}
F((u, i, \lambda)(\alpha, j, \mu)) & =F(u \alpha, \Phi(u, i, \lambda)(j), \Psi(\alpha, j, \mu)(\lambda)) \\
& =\left(u \alpha, \theta_{R}(\Phi(u, i, \lambda)(j)), \theta_{L}(\Psi(\alpha, j, \mu)(\lambda))\right) .
\end{aligned}
$$

Since $F$ is a homomorphism, we have

$$
\begin{aligned}
F((u, i, \lambda)(\alpha, j, \mu)) & =\left(u, \theta_{R}(i), \theta_{L}(\lambda)\right)\left(\alpha, \theta_{R}(j), \theta_{L}(\mu)\right) \\
= & \left(u \alpha, \Phi\left(u, \theta_{R}(i), \theta_{L}(\lambda)\right)\left(\theta_{R}(j)\right), \Psi\left(\alpha, \theta_{R}(J), \theta_{L}(\mu)\right)\left(\theta_{L}(\lambda)\right)\right) .
\end{aligned}
$$

Hence we get $\theta_{R}(\Phi(u, i, \lambda)(j))=\Phi\left(u, \theta_{R}(i), \theta_{L}(\lambda)\right)\left(\theta_{R}(j)\right)$. The second condition is proved dually.

Conversely if $\theta_{R}: I \rightarrow I^{\prime}, \theta_{L}: \Lambda \rightarrow \Lambda^{\prime}$ are mappings satisfying the given conditions, then the mapping $F$ defined above is clearly a morphism of coextensions if $F$ is a homomorphism. To prove this, take $(u, i, \lambda),(v, j, \mu) \in S$. Then

$$
\begin{gathered}
F((u, i, \lambda)(v, j, \mu))=F(u v, \Phi(u, i, \lambda)(j), \Psi(v, j, \mu)(\lambda)) \\
=\left(u v, \theta_{R}(\Psi(u, i, \lambda)(j)), \theta_{L}(\Psi(v, j, \mu)(\lambda))\right), \\
\begin{aligned}
F(u, i, \lambda) F(v, j, \mu)=\left(u, \theta_{R}(i), \theta_{L}(\lambda)\right)\left(v, \theta_{R}(j), \theta_{L}(\mu)\right) \\
=\left(u v, \Phi\left(u, \theta_{R}(i), \theta_{L}(\lambda)\right)\left(\theta_{R}(j)\right), \Psi\left(v, \theta_{R}(j), \theta_{L}(\mu)\right)\left(\theta_{L}(\lambda)\right)\right) .
\end{aligned}
\end{gathered}
$$

The given conditions imply that $F$ is a homomorphism.

3. Normal coextensions. A coextension $(E, \theta)$ of a biordered set $F$ is said to be normal if for $e \in E$ and $\beta \in F$ with $\beta \omega e \theta$, there exists a unique $f \in E$ with $f \omega e$ and $f \theta=\beta$. In [5] is was shown that such coextensions are coextensions by rectangular biordered sets and may be described in terms of two set valued functors 
$\chi_{R}: I=F / \Re \rightarrow$ Set and $\chi_{L}: \Lambda=F / \mathcal{L} \rightarrow$ Set and we shall write $(E, \theta)=$

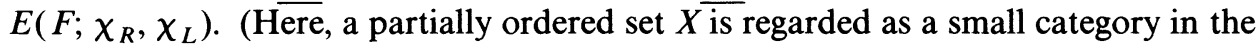
usual way; with morphisms from $x$ to $y$ when $y \leqslant x$.) Theorems 2.2 and 2.3 can be simplified considerably when the coextensions of biordered sets considered are normal.

LeMMA 3.1. Let $(E, \theta)$ be a normal coextension of the biordered set $F$. Then for all $e \in E, \theta_{e}=\theta \mid \omega(e)$ is an isomorphism of $\omega(e)$ onto $\omega(e \theta)$.

PROof. It is clear that $\theta_{e}$ is a surjective bimorphism of $\omega(e)$ onto $\omega(e \theta)$. To prove that $\theta_{e}$ is an isomorphism, it is sufficient to show that $\theta_{e}$ is one-to-one. This is obvious from the definition of normality.

TheOREM 3.2. Let $(E, \theta)$ be a normal coextension of the biordered set $F$. Then we have the following.

(a) $\bar{\theta}$ is a homomorphism of $T(E)$ onto $T(F)$.

(b) $\operatorname{ker} \bar{\theta}=\eta_{\theta} \subseteq \rho(T(E))$.

Proof. To prove (a), it is sufficient to show that $\mathcal{T}_{\theta}=T^{*}(E)$ and $\operatorname{im} \theta^{*}=T^{*}(F)$; so, let $\alpha \in T^{*}(E)$. Then by Lemma 3.1, $\alpha^{\prime}=\theta_{e_{\alpha}}^{-1} \circ \alpha \circ \theta_{f_{\alpha}}$ is an isomorphism of $\omega\left(e_{\alpha} \theta\right)$ onto $\omega\left(f_{\alpha} \theta\right)$ such that $\theta \circ \alpha^{\prime}=\alpha \circ \theta$. Thus $\theta^{*}(\alpha)=\alpha^{\prime}$ and $\alpha \in \mathcal{T}_{\theta}$. Therefore $\sigma_{\theta}=T^{*}(E)$. Similarly if $\alpha^{\prime} \in T^{*}(F)$, and if $e, f \in E$ with $e \theta=e_{\alpha^{\prime}}$ and $f \theta=f_{\alpha^{\prime}}$, then $\alpha=\theta_{e} \circ \alpha^{\prime} \circ \theta_{f}^{-1} \in T^{*}(E)$ and $\theta^{*}(\alpha)=\alpha^{\prime}$. Thus im $\theta^{*}=T^{*}(f)$. This proves (a).

Since, always, $\eta_{\theta} \subseteq \operatorname{ker} \bar{\theta}$, the statement (b) will follow if we show that $\operatorname{ker} \bar{\theta} \subseteq$ $\rho(T(E))$; or equivalently, $\bar{\theta}$ is one-to-one on the $\mathcal{G}$-classes of $T(E)$. Let $\bar{\alpha}, \bar{\beta}$ be $\mathcal{H}$-equivalent elements in $T(E)$, where $\alpha, \beta \in T^{*}(E)$. If $\bar{\alpha} \bar{\theta}=\bar{\beta} \bar{\theta}$, then $e_{\alpha} \Re e_{\beta}$ and $f_{\alpha} \mathcal{L} f_{\beta}$ and so $\beta_{1}=\tau\left(e_{\alpha}, e_{\beta}\right) \beta \tau\left(f_{\beta}, f_{\alpha}\right) p \beta$ (see [6]) and $\beta_{1}, \alpha \in T^{*}(E)\left(e_{\alpha}, f_{\alpha}\right)$. So, $\bar{\beta}_{1} \bar{\theta}=\bar{\beta} \bar{\theta}=\bar{\alpha} \bar{\theta}$ and so $\theta^{*}\left(\beta_{1}\right) p \theta^{*}(\alpha)$. Since $\theta^{*}\left(\beta_{1}\right), \theta^{*}(\alpha) \in T^{*}(F)\left(e_{\alpha} \theta, f_{\alpha} \theta\right)$, it follows that $\theta^{*}\left(\beta_{1}\right)=\theta^{*}(\alpha)$. Thus

$$
\alpha=\theta_{e_{\alpha}} \circ \theta^{*}(\alpha) \circ \theta_{f_{\alpha}}^{-1}=\theta_{e_{\alpha}} \circ \theta^{*}\left(\beta_{1}\right) \circ \theta_{f_{\alpha}}^{-1}=\beta_{1} .
$$

So $\bar{\alpha}=\bar{\beta}_{1}=\bar{\beta}$.

We have observed before that if $(E, \theta)$ is a strong coextension of $F$ by rectangular biordered sets and if $U$ is a regular semigroup with $E(U)=F$, it may not be possible always to find a coextension of $U$ by rectangular bands inducing $(E, \theta)$ as the biordered set (see the remark after Theorem 2.2). By Theorem 2.2, a necessary and sufficient condition for the existence of such a coextension is that there exists an idempotent separating homomorphism of $U$ into $S_{\theta}$. If $(E, \theta)$ is normal, the foregoing theorem implies that $S_{\theta}$ is isomorphic to $T(F)$. Since $\eta_{\theta} \subseteq \rho(T(E)),(E, \theta)$ is a strong coextension of $F$ and so, the existence of the natural homomorphism $\overline{\mathrm{a}}_{S}$ : $U \rightarrow T(F)$ implies that $U$ satisfies the condition of Theorem 2.2. Thus we have

Corollary 3.3. Let $(E, \theta)$ be a normal coextension of a biordered set $F$. If $U$ is any regular semigroup with $E(U)=F$, then there exists a coextension of $U$ by rectangular bands inducing $(E, \theta)$. In particular every normal coextension of $F$ is strong relative to every idempotent-generated regular semigroup $S^{\prime}$ with $E\left(S^{\prime}\right)=F$. 
For convenience we shall say that a coextension of a regular semigroup by rectangular bands is normal if it induces a normal coextension on its biordered sets. Given a regular semigroup $U$, the subcategory of the category $\underline{R}(U)$ consisting of all normal coextensions will be denoted by $\underline{N} \underline{R}(U)$. The following theorem describes the construction of objects and morphisms of $\underline{N} \underline{R}(U)$.

If $E\left(F ; \chi_{R}, \chi_{L}\right)$ is a normal coextension of $F$ and if $U$ is any regular semigroup with $E(U)=F$, in the following, it is convenient to identify $I=F / \Re$ with $U / \mathcal{R}$ and $\Lambda=F / \mathcal{L}$ with $U / \mathcal{L}$ so that $\chi_{R}$ and $\chi_{L}$ are functors on $U / R=I$ and $U / \mathcal{L}=\Lambda$ respectively.

THEOREM 3.4. (1) Let $(E, \theta)=E\left(F ; \chi_{R}, \chi_{L}\right)$ be a normal coextension of the biordered set $F$ and let $U$ be a regular semigroup with $E(U)=F$. Then

$$
S=\left\{(u, i, \lambda): u \in U, i \in \chi_{R}\left(R_{u}\right), \lambda \in \chi_{L}\left(L_{u}\right)\right\}
$$

with product defined by

$$
(u, i, \lambda)(v, j, \mu)=\left(u v, i \chi_{R}\left(R_{u}, R_{u v}\right), \mu \chi_{L}\left(L_{v}, L_{u v}\right)\right)
$$

is a regular semigroup and the mapping $p:(u, i, \lambda) \rightarrow u$ is a homomorphism of $S$ onto $U$ such that $(S, p)=S\left(U ; \chi_{R}, \chi_{L}\right)$ is a normal coextension of $U$. Further the map $\pi$ : $(\alpha, i, \lambda) \rightarrow(i, \lambda)$ is an isomorphism of $E(S)$ onto $E$ such that $E(p)=\pi \circ \theta$.

Conversely if $(S, p)$ is any normal coextension of $U$ define $\chi_{R}$ by $\chi_{R}\left(R_{u}\right)=\left\{R_{x}\right.$ : $x p=u$ \} for $R_{u} \in I$ and for $R_{u}, R_{v} \in I$ with $R_{v} \leqslant R_{u}$ and $R_{x} \in \chi_{R}\left(R_{u}\right)$, define $R_{x} \chi_{R}\left(R_{u}, R_{v}\right)=R_{y}$ where $R_{y}$ is the unique element in $\chi_{R}\left(R_{v}\right)$ such that $R_{y} \leqslant R_{x}$. Then $\chi_{R}$ is a set-valued functor on I. Dually

$$
X_{L}\left(L_{u}\right)=\left\{L_{x}: x p=u\right\}, \quad L_{u} \in \Lambda,
$$

and $L_{x} \chi_{L}\left(L_{u}, L_{v}\right)=L_{y}$ where $L_{v} \leqslant L_{u}$ and $L_{y}$ is the unique element in $\chi_{L}\left(L_{v}\right)$ such that $L_{y} \leqslant L_{x}$, defines a set-valued functor on $\Lambda$. Moreover, the map $t: x \rightarrow\left(x p, R_{x}, L_{x}\right)$ is an isomorphism of $(S, p)$ onto $S\left(U ; \chi_{R}, \chi_{L}\right)$.

(2) Let $\phi: S\left(U ; \chi_{R}, \chi_{L}\right) \rightarrow S\left(U ; \chi_{R}^{\prime}, X_{L}^{\prime}\right)$ be a morphism of normal coextensions. Then there exist natural transformations

$$
\sigma: \chi_{R} \rightarrow \chi_{R}^{\prime}, \quad \tau: \chi_{L} \rightarrow \chi_{L}^{\prime}
$$

such that for all $(u, i, \lambda) \in S\left(U ; \chi_{R}, \chi_{L}\right)$,

$$
(u, i, \lambda) \phi=\left(u, i \sigma_{R_{u}}, \lambda \tau_{L_{u}}\right) .
$$

Conversely, given two natural transformations $\sigma: \chi_{R} \rightarrow \chi_{R}^{\prime}$ and $\tau: \chi_{L} \rightarrow \chi_{L}^{\prime}$, the map $\phi$ defined by the equation above is a morphism of the coextensions: $\phi$ is an isomorphism if and only if $\sigma$ and $\tau$ are natural isomorphisms.

Proof. (1) Let $(u, i, \lambda),(v, j, \mu),(w, k, \nu) \in S$. Then by the definition of product in $S$,

$$
\begin{aligned}
& {[(u, i, \lambda)(v, j, \mu)](w, k, \nu)} \\
& \quad=\left(u v w, i \chi_{R}\left(R_{u}, R_{u v}\right) \chi_{R}\left(R_{u v}, R_{u v w}\right), \nu \chi_{L}\left(L_{w}, L_{u v w}\right)\right)
\end{aligned}
$$


and

$$
\begin{aligned}
& (u, i, \lambda)[(v, j, \mu)(w, k, \nu)] \\
& \quad=\left(u v w, i \chi_{R}\left(R_{u}, R_{u v w}\right), \nu \chi_{L}\left(L_{w}, L_{v w}\right) \chi_{L}\left(L_{v w}, L_{u v w}\right)\right) .
\end{aligned}
$$

Since $\chi_{R}$ and $\chi_{I}$ are functors we have

$$
\begin{gathered}
\chi_{R}\left(R_{u}, R_{u v}\right) \chi_{R}\left(R_{u v}, R_{u v w}\right)=\chi_{R}\left(R_{u}, R_{u v w}\right), \\
\chi_{L}\left(L_{w}, L_{v w}\right) \chi_{L}\left(L_{v w}, L_{u v w}\right)=\chi_{L}\left(L_{w}, L_{u v w}\right)
\end{gathered}
$$

and so the product defined in $S$ is associative. Further, for $(u, i, \lambda) \in S$, choose $u^{\prime} \in V(u), j \in \chi_{R}\left(R_{u^{\prime}}\right)$ and $\mu \in \chi_{L}\left(L_{u^{\prime}}\right)$. Since $R_{u}=R_{u u^{\prime}}$ and $L_{u^{\prime}}=L_{u u^{\prime}}$ it follows that

$$
\begin{aligned}
i \chi_{R}\left(R_{u}, R_{u u^{\prime}}\right) & =i \chi_{R}\left(R_{u}, R_{u}\right)=i, \\
\mu \chi_{L}\left(L_{u^{\prime}}, L_{u u^{\prime}}\right) & =\mu \chi_{L}\left(L_{u^{\prime}}, L_{u^{\prime}}\right)=\mu
\end{aligned}
$$

and so, $(u, i, \lambda)\left(u^{\prime}, j, \mu\right)=\left(u u^{\prime}, i, \mu\right)$. Hence

$$
\begin{aligned}
(u, i, \lambda)\left(u^{\prime}, j, \mu\right)(u, i, \lambda) & =\left(u u^{\prime}, i, \mu\right)(u, i, \lambda) \\
& =\left(u, i \chi_{R}\left(R_{u u^{\prime}}, R_{u}\right), \lambda \chi_{L}\left(L_{u}, L_{u}\right)(\lambda)\right)=(u, i, \lambda) .
\end{aligned}
$$

Similarly $\left(u^{\prime}, j, \mu\right)(u, i, \lambda)\left(u^{\prime}, j, \mu\right)=\left(u^{\prime}, j, \mu\right)$. Hence $\left(u^{\prime}, j, \mu\right) \in V((u, i, \lambda))$ and so $S$ is regular. From the definition of product it is clear that the map $p$ : $(u, i, \lambda) \rightarrow u$ is a homomorphism and that, for any $\alpha \in F, \alpha p^{-1}$ is a rectangular subband of $E(S)$. Also $(u, i, \lambda) \in E(S)$ if and only if $u \in F$ and $(i, \lambda) \in \chi_{R}\left(R_{u}\right)$ $\times \chi_{L}\left(L_{u}\right)=E_{u}$. Therefore the mapping $\pi:(\alpha, i, \lambda) \rightarrow(i, \lambda)(\alpha \in F)$ is a bijection of $E(S)$ onto $E$ such that $\pi \circ \theta=E(p)$, where $\theta$ is the bimorphism associated with the coextension $E\left(F, \chi_{R}, \chi_{L}\right)$ which sends $(i, \lambda) \in E$ to $\alpha$. Moreover, by the definition of product in $S$ and basic product in $E, \pi$ is an isomorphism (see [5, Theorem 5.5]).

Conversely assume that $(S, p)$ is a normal coextension of $U$. It follows from the definition of normality that for all $R_{x} \in \chi_{R}\left(R_{u}\right)$, there exists a unique $R_{y} \in \chi_{R}\left(R_{v}\right)$ (where $R_{u}, R_{v} \in I$ and $R_{v} \leqslant R_{u}$ ) such that $R_{y} \leqslant R_{x}$. Thus the definition of $\chi_{R}\left(R_{u}, R_{v}\right)$ is valid. It is also easy to see that $\chi_{R}$ is a functor. Dually $\chi_{L}$ is also a functor. The map $t$ sending $x$ to $\left(x p, R_{x}, L_{x}\right)$ is clearly single-valued. Also since $(S, p)$ is a coextension by rectangular bands, $t$ is a bijection. To prove that $t$ is an isomorphism, we first observe that $R_{x y} \leqslant R_{x}$ and $L_{x y} \leqslant L_{y}$ for all $x, y \in S$. Hence if $x p=u, y p=v$,

$$
R_{x} \chi_{R}\left(R_{u}, R_{u v}\right)=R_{x y}, \quad L_{y} \chi_{L}\left(L_{v}, L_{u v}\right)=L_{x y}
$$

and so $(x t)(y t)=\left((x y) p, R_{x y}, L_{x y}\right)=(x y) t$. If $p^{\prime}$ is the projection of $S\left(U ; \chi_{R}, \chi_{L}\right)$ onto $U$, it is clear from the definition of $t$ that $p=t \circ p^{\prime}$ and so $t$ is an isomorphism of coextensions.

(2) For each $R_{u} \in I$ and $i \in \chi_{R}\left(R_{u}\right), \lambda \in \chi_{L}\left(L_{u}\right),\left(u, i \sigma_{R_{u}}, \lambda \tau_{L_{u}}\right) \in S\left(U ; \chi_{R}^{\prime}, \chi_{L}^{\prime}\right)$ if and only if $i \sigma_{R_{u}} \in \chi_{R}^{\prime}\left(R_{u}\right)$ and $\lambda \tau_{L_{u}} \in \chi^{\prime}\left(L_{u}\right)$. Thus $\phi$, defined by

$$
(u, i, \lambda) \phi=\left(u, i \sigma_{R_{u}}, \lambda \tau_{L_{u}}\right) \text {, }
$$


is a mapping of $S\left(U ; \chi_{R}, \chi_{L}\right)$ into $S\left(U ; \chi_{R}^{\prime}, \chi_{L}^{\prime}\right)$ if and only if $\sigma_{R_{u}}$ is a mapping of $\chi_{R}\left(R_{u}\right)$ into $\chi_{R}^{\prime}\left(R_{u}\right)$ and $\tau_{L_{u}}$ is a mapping of $\chi_{L}\left(L_{u}\right)$ into $\chi_{L}^{\prime}\left(L_{u}\right)$. Also

$$
((u, i, \lambda)(v, j, \mu)) \phi=\left(u v, i \chi_{R}\left(R_{u}, R_{u v}\right) \sigma_{R_{u v}}, \mu \chi_{L}\left(L_{v}, L_{u v}\right) \tau_{L_{u v}}\right)
$$

and

$$
(u, i, \lambda) \phi(v, j, \mu) \phi=\left(u v, i \sigma_{R_{u}} \chi_{R}\left(R_{u}, R_{u v}\right), \mu \tau_{L_{v}} \chi_{L}^{\prime}\left(L_{v}, L_{u v}\right)\right)
$$

Therefore $\phi$ is a homomorphism if and only if

$$
i \chi_{R}\left(R_{u}, R_{u v}\right) \sigma_{R_{u v}}=i \sigma_{R_{u}} \chi_{R}^{\prime}\left(R_{u}, R_{u v}\right)
$$

and

$$
\mu \chi_{L}\left(L_{v}, L_{u v}\right) \tau_{L_{u v}}=\mu \tau_{L_{v}} \chi_{L}^{\prime}\left(L_{v}, L_{u v}\right)
$$

that is, if and only if $\sigma: R_{u} \rightarrow \sigma_{R_{u}}$ and $\tau: L_{u} \rightarrow \tau_{L_{u}}$ are natural transformations. It is also clear that $\phi$ is a bijection if and only if, for each $R_{u} \in I, \sigma_{R_{u}}$ and $\tau_{L_{u}}$ are bijections. Therefore $\phi$ is an isomorphism if and only if both $\sigma$ and $\tau$ are natural isomorphisms.

Let $[I$, Set] denote the category of all functors from $I=U / \Re$ into Set. Objects in $[I$, Set] are set-valued functors on $I$ and morphisms are natural transformations. If $\sigma^{1}: \overline{\chi_{1}} \rightarrow \chi_{2}$ and $\sigma^{2}: \chi_{2} \rightarrow \chi_{3}$ are morphisms in [I, Set], the composition of $\sigma^{1}$ and $\sigma^{2}$ is the natural transformation that sends $R_{u}$ to $\sigma_{R_{u}}^{1} \bar{\circ}_{R_{u}}^{2}$. Now consider the category $[I$, Set $] \times[\Lambda$, Set $]$. Then it is easy to see that the maps that send $\left(\chi_{R}, \chi_{L}\right) \in[I$, Set $]$ $\times \overline{[\Lambda}$, Set $]$ to $\bar{S}\left(U ; \chi_{R}, \chi_{L}\right)$ and pairs of natural transformations $(\sigma, \tau)$ to $\overline{\text { the }}$ homomorphism $\phi=S(U ; \sigma, \tau)$ defined in the foregoing theorem define a functor $S(U ;$,$) of [I$, Set $] \times[\Lambda$, Set $]$ into the category $N R(U)$. Further, $\chi:(S, p) \rightarrow\left(\chi_{R}, \chi_{L}\right)$, $\phi \rightarrow(\sigma, \tau)$ where $\left(\chi_{R}, \overline{\chi_{L}}\right)$ and $(\sigma, \tau)$ are pairs of functors and natural transformations defined in the foregoing theorem, is also a functor of $N R(U)$ to $[I, \operatorname{Set}] \times$ $[\Lambda$, Set $]$ such that $S(U ;,) \circ \chi$ is naturally isomorphic to $\iota_{[I, \text { Set }] \times[\Lambda, \text { Set }]}$ and $\left.\chi \circ \overline{S(U ;} ;,\right)$ is naturally isomorphic to $\iota_{N R(U)}$. Thus

THEOREM 3.5. The category $N R(U)$ of all normal coextensions of a regular semigroup $U$ is naturally equivalent to $[I$, Set $] \times[\Lambda$, Set $]$ where $I=U / R$ and $\Lambda=U / \varrho$.

The foregoing can be easily modified to show that the category of all normal coextensions of a biordered set $F$ is naturally equivalent to the product category $[F / \Re$, Set $] \times[F / \mathcal{L}$, Set $]$.

\section{REFERENCES}

1. A. H. Clifford and G. B. Preston, The algebraic theory of semigroups, Math. Surveys, no. 7, Amer. Math. Soc., Providence, R. I., vol. 1, 1961; vol. 2, 1967.

2. P. A. Grillet, Structure of regular semigroups. I, II, III, Semigroup Forum 8 (1974), 177-183; 254-259; 260-265.

3. T. E. Hall, Orthodox semigroups, Pacific J. Math 39 (1971), 677-686.

4. Congruences and Green's relations on regular semigroups, Glasgow Math. J. 13 (1972), $167-175$ 
5. J. Meakin and K. S. S. Nambooripad, Coextensions of regular semigroups by rectangular bands. I, Trans. Amer. Math. Soc. 269 (1982), 197-224.

6. K. S. S. Nambooripad, Structure of regular semigroups. I, Mem. Amer. Math. Soc. no. 224 (1979).

7. __ Relations between cross-connections and biordered sets, Semigroup Forum 16 (1978), 67-81.

8. N. R. Reilly and H. E. Scheiblich, Congruences on regular semigroups, Pacific J. Math. 23 (1967), 349-360.

9. M. Yamada, Regular semigroups whose idempotents satisfy permutation identities, Pacific J. Math. 21 (1967), 371-392.

Department of Mathematics and Statistics, University of Nebraska, Lincoln, Nebraska 68588

Department of Mathematics, University of Kerala, Kariavattom 695581 India 